\title{
Effect of Prior Bilateral Oophorectomy on the Presentation of Breast Cancer in BRCA1 and BRCA2 Mutation Carriers
}

\author{
Kelly A. Metcalfe ${ }^{1,2}$, William D. Foulkes ${ }^{3}$, Henry T. Lynch ${ }^{4}$, Parviz Ghadirian ${ }^{5}$, Nadine Tung ${ }^{6}$, Ivo A. Olivotto ${ }^{7}$, Ellen Warner ${ }^{8}$, \\ Olufunmilayo Olopade', Andrea Eisen ${ }^{8}$, Barbara Weber ${ }^{10}$, Jane McLennan" ${ }^{11}$ Ping Sun', Steven A. Narod ${ }^{2}$ \\ 'Faculty of Nursing, University of Toronto, Canada; 2The Centre for Research in Women's Health, Sunnybrook and Women's College Health Sciences Centre, Toronto, \\ Canada; ${ }^{3}$ Program in Cancer Genetics, McGill University, Montreal, Canada; ${ }^{4}$ Department of Preventive Medicine and Public Health, Creighton University School of \\ Medicine, Omaha, USA; 5 Epidemiology Research Unit, Research Centre of Centre Hospitalier de l'Université de Montréal, Montreal, Canada; 6 Beth Israel Deaconess \\ Hospital, Boston Massachusetts, USA; ${ }^{7 B C}$ Cancer Agency, Victoria, B.C., Canada; ${ }^{8}$ Sunnybrook and Women's College Health Sciences Centre, Toronto, Canada; \\ ?University of Chicago, USA; 10Department of Hematology/Oncology, University of Pennsylvania, Philadelphia, USA; "1'University of California, San Francisco, USA
}

Key words: BRCA1, BRCA2, breast cancer, oophorectomy

Corresponding author: Dr. Steven A. Narod, Canada Research Chair in Breast Cancer, Centre for Research in Women's Health, 790 Bay Street, 7th floor, Toronto, ON M5G 1N8 Canada. Phone +416 351 3765, fax +416 351 3767, e-mail: steven.narod@swchsc.on.ca

Submitted: 7 April 2005

Accepted: 28 April 2005

\begin{abstract}
Purpose: To compare the presentation of invasive breast cancer in BRCA1 and BRCA2 mutation carriers with and without prior bilateral oophorectomy.

Patients and methods: Women with a BRCA1 or BRCA2 mutation with the diagnosis of invasive breast cancer were identified from ten cancer genetics clinics. The medical history, medical treatment records and pathology reports for the breast cancers were reviewed. Information was abstracted from medical charts, including history (and date) of oophorectomy, date of breast cancer diagnosis, stage of disease, and pathologic characteristics of the breast cancer. Women with prior bilateral oophorectomy were matched by age, year of diagnosis, and mutation with one or more women who had two intact ovaries at the time of breast cancer diagnosis. Characteristics of the breast tumours were compared between the two groups. Results: Women with prior bilateral oophorectomy presented with smaller tumours on average compared to women without prior oophorectomy (mean size $1.50 \mathrm{~cm}$ vs. $1.95 \mathrm{~cm} ; \mathrm{p}=0.01$ ). Additionally, although not statistically significant, women with intact ovaries were more likely to have high-grade tumour $(70 \%$ vs. $54 \%: p=0.10)$ and to have positive lymph nodes ( $34 \%$ vs. $18 \% ; p=0.11$ ) compared to women with prior bilateral oophorectomy.

Conclusions: Bilateral oophorectomy prior to breast cancer appears to favourably influence the biological presentation of breast cancer in BRCA1 and BRCA2 mutation carriers.
\end{abstract}

\section{Introduction}

Women with a BRCA1 or BRCA2 mutation have up to an $87 \%$ lifetime risk of developing invasive breast cancer [1]. The surgical removal of the ovaries in women with a BRCA1 or BRCA2 mutation has been shown to reduce the risk of developing breast cancer
[2]. Nevertheless, some women who have had bilateral oophorectomy go on to develop breast cancer. Previous research has suggested that there may be an association between menopausal status and prognostic features of breast cancer [3], but this association has not been studied in women with a BRCA1 or BRCA2 mutation. 
Women with a BRCA1 or BRCA2 mutation are encouraged to consider the option of prophylactic oophorectomy to prevent both breast and ovarian cancers. The recommended age for surgery is typically prior to the age at which natural menopause would occur. In our recent study of BRCA1 and BRCA2 mutation carriers without cancer, $67 \%$ of women had prophylactic oophorectomy to prevent breast and ovarian cancer [4]. Bilateral oophorectomy reduces the risk of developing breast cancer by up to $53 \%$ in BRCA1 and BRCA2 mutation carriers [2]. It has also been shown that ovarian ablation for the treatment of early breast cancer significantly improves survival [5]. However, it is unknown if prior bilateral oophorectomy reduces breast cancer mortality in women with a BRCA1 or BRCA2 mutation. If oophorectomy selectively prevents less aggressive breast tumours, then the observed reduction in incidence possibly may not be accompanied by parallel reduction in mortality. It is therefore of interest to know whether prior oophorectomy has any influence on the biological presentation of breast cancer. We examined pathologic features of invasive breast cancer in a series of women with a BRCA1 or BRCA2 mutation and compared features in women with and without prior bilateral oophorectomy.

\section{Methods}

\section{Study subjects}

Pedigrees of cancer families followed at ten participating cancer genetics clinics were reviewed. A family was considered to be eligible for the study when a BRCA1 or BRCA2 mutation was documented in the family and at least one case of invasive breast cancer was recorded. Eligible study subjects included all women from these families who were diagnosed with Stage I or Stage II breast cancer at the age of 65 or below, from 1975 to 2000. Living and deceased women were eligible, but those with prior diagnosis of cancer (including ovarian cancer) or those who resided outside of North America were excluded. It was not necessary that the woman was proven carrier of the mutation found in the family to be included in the study; however affected women who were known to be non-carriers were excluded.

We identified a total of 1139 breast cancer cases in 337 families. Pedigree review indicated that 647 of these were currently alive and that 492 were deceased. Of the total of 1139 cases of breast cancer, 320 women were excluded because the date of diagnosis was before 1975 and 50 women were excluded because the age of diagnosis was above 65 years. An additional 19 women were non-carriers of the familial mutation and were therefore excluded. Ten women were excluded because they had cancer (including ovarian) diagnosed prior to breast cancer, and eleven women were excluded because they were treated outside of North America.

We were able to obtain identifying information for 587 of the remaining 729 women (80.5\%). An attempt was made to contact each of these or her next of kin to obtain permission to review the medical records. Thirteen women (or their next of kin) refused to give their consent to the release of the medical records. The medical record was requested from the hospital where treatment was received for the remaining 574 women. In 54 cases, the hospital was not able to locate the record or did not forward the requested documents. The medical record was obtained on the remaining 520 women (91\%).

After review of the medical records an additional 29 women were excluded. Of these, 22 women were excluded because the tumour was stage three or four; six women were excluded because the tumour was noninvasive (DCIS or LCIS); and one woman was excluded because she refused treatment. The remaining 491 women were included in the analysis.

\section{Study protocol}

The medical treatment records and pathology documents were reviewed. Information that was abstracted from the medical charts included date of breast cancer diagnosis, size of invasive tumour, nodal status (number of nodes positive, number of nodes examined), nuclear grade, ER (estrogen receptor) status, PR (progesterone receptor) status, oophorectomy (yes/no, date, indication), and current vital status (living/deceased, cause of death).

\section{Statistical analysis}

All data were analysed using the SAS software program (version 8.2). Mean values of continuous variables of cases and controls were compared by t-test and frequencies of categorical variables were compared by Chi-square test. In the comparison of variables, the subjects with missing data were excluded. In the control sets, if all the subjects had no data, it was listed as missing data, or else the mean value of the control set was used as the control mean for that case.

\section{Results}

There were 491 women who were treated for stage I or II breast cancer between 1975 and 2000. Sixteen 
Table 1. Comparison of cases and controls

\begin{tabular}{|c|c|c|c|}
\hline Variables & $\begin{array}{c}\text { Controls (no bilateral oophorectomy) } \\
\text { (27 sets, } 138 \text { subjects) }\end{array}$ & $\begin{array}{l}\text { Cases (had bilateral oophorectomy) } \\
\qquad(\mathrm{N}=27)\end{array}$ & $P$ \\
\hline mean $\mathrm{DOB}$ & 1943.8 & 1943.4 & $0.86^{*}$ \\
\hline mean age of diagnosis (range) & $47.9(35.7-63.3)$ & $48.0(36.0-63.2)$ & $0.96^{*}$ \\
\hline \multicolumn{4}{|l|}{ mutation } \\
\hline BRCA1 & $74.1 \%$ & $74.1 \%$ & \\
\hline BRCA2 & $25.9 \%$ & $25.9 \%$ & \\
\hline $\begin{array}{l}\text { ER-status (pos \%) } \\
\text { missing (n) }\end{array}$ & $\begin{array}{c}33.6 \\
5\end{array}$ & $\begin{array}{c}40.0 \\
7\end{array}$ & $0.62^{*}$ \\
\hline $\begin{array}{l}\text { size }(\mathrm{cm}) \text { (range) } \\
\text { missing }(\mathrm{n})\end{array}$ & $\begin{array}{c}1.95(1.0-3.0) \\
1\end{array}$ & $\begin{array}{c}1.50(0.2-3.2) \\
1\end{array}$ & $0.01^{*}$ \\
\hline nodal status (\% positive) & 33.7 & 18.5 & $0.11^{*}$ \\
\hline
\end{tabular}

subjects had data missing on oophorectomy or date of oophorectomy, and therefore were not included in the analysis. An additional five women had a bilateral oophorectomy within six months prior to breast cancer diagnosis, and they were also excluded. Cases were identified as those who had bilateral oophorectomy at least six months prior to being diagnosed with invasive breast cancer. There were 28 women who were identified as cases. These subjects were matched with the remaining 442 control subjects by (1) date of birth within five years; (2) breast cancer diagnosis within one year; and (3) mutation (BRCA1 or BRCA2). There were no controls found for one case, and therefore it was excluded. For 27 cases at least one control was found, resulting in a total of 138 controls. The ratio of case to control was 1:4.8 (27:138).

The mean age of breast cancer diagnosis for all of the subjects (both cases and controls) was 47.9 years (range 35.7-63.3 years). The mean time between oophorectomy and breast cancer diagnosis for the cases was 7.5 years $(S D=6.8)$.

Breast tumour characteristics (including ER status, nuclear grade, size, and nodal status) of the cases and controls were compared (Table 1). There was no difference in ER status of the breast cancers for the women who had bilateral oophorectomy compared to those with intact ovaries (40.0\% vs. 33.6\% ER-positive; $p=0.62$ ). There was a significant difference in the tumour size between the two groups of subjects. Women with prior bilateral oophorectomy presented with smaller tumours on average compared to those without oophorectomy $(1.50 \mathrm{~cm}$ vs. $1.95 \mathrm{~cm} ; \mathrm{p}=0.01)$. $23.1 \%$ of women with prior oophorectomy had tumours of $1 \mathrm{~cm}$ or less, compared to only $3.9 \%$ of women with intact ovaries $(O R=7.50 ; p=0.04)$. Additionally (although not statistically significant) women with prior oophorectomy were more likely to have a lower grade tumour (grade I or II) $(p=0.10)$ with negative lymph nodes $(p=0.11)$. Seventy percent of women with intact ovaries presented with a poorly differentiated (grade III) tumour compared to $54 \%$ of those with prior bilateral oophorectomy $(p=0.10)$. Additionally, women with intact ovaries were almost twice as likely to present with positive lymph nodes compared to women without ovaries (33.7\% vs. 18.5\%; $p=0.11)$.

Tumour characteristics were also examined by gene type (BRCA1 vs. BRCA2). BRCA2 carriers with prior oophorectomy presented with lower grade tumours than women with BRCA2 mutation with intact ovaries $(p=0.04)$. This was not observed in women with a BRCA1 mutation (Table 2). The analyses were also performed for ER status, size, and nodal status. No differences between BRCA1 and BRCA2 mutation carriers were observed (data not presented).

Women were dichotomized by tumour characteristics that were associated with a poor and good prognosis. Women with a better prognosis were classified as those with grade I or grade II tumour and tumour size of less than or equal to $2 \mathrm{~cm}$. All other subjects were assigned to the group that had less favourable tumour characteristics. Women with prior oophorectomy were twice as likely to have tumours that were classified as having a better prognosis than women with intact ovaries $(\mathrm{OR}=0.50 ; 95 \% \mathrm{Cl} 0.17-1.47 ; \mathrm{p}=0.21)$.

\section{Discussion}

This is the first study to examine the influence of prior bilateral oophorectomy on the presentation of 
Table 2. Comparison of grade by mutation (BRCA1 and BRCA2)

\begin{tabular}{lcccc}
\hline Mutation & Grade & $\begin{array}{c}\text { Controls } \\
\text { (no bilateral oophorectomy) } \\
(27 \text { sets, } 138 \text { subjects) }\end{array}$ & $\begin{array}{c}\text { Cases } \\
\text { (had bilateral oophorectomy) } \\
(\mathbf{N}=27)\end{array}$ & $P$ \\
\hline BRCA1 & 1 & $2(2 \%)$ & $2(10.5 \%)$ & $0.10^{* *}$ \\
and BRCA2 & 2 & $28(28 \%)$ & $7(36.8 \%)$ & $10(53.6 \%)$ \\
& 3 & $70(70 \%)$ & 8 & 0.62 \\
\hline BRCA1 & missing & 38 & $1(6.7 \%)$ & \\
& 1 & $2(2.2 \%)$ & $1(26.7 \%)$ & 0.04 \\
\hline BRCA2 & 2 & $26(28.3 \%)$ & $1(25.0 \%)$ & $3(75.0 \%)$ \\
\end{tabular}

** In this test the data in control were not the mean of set.

invasive breast cancer in BRCA1 and BRCA2 mutation carriers.

We matched women with prior bilateral oophorectomy to those with intact ovaries in order to compare the prognostic features of breast cancer. Although the sample size was small, we observed that women with prior bilateral oophorectomy were more likely to be diagnosed with smaller, node negative, lower grade tumours. There did not seem to be an influence of oophorectomy on ER status, suggesting that oophorectomy prevents ER-positive and ER-negative tumours equally. Our results suggest that if breast cancer develops after bilateral oophorectomy, the prognostic features are likely to be more favourable than for those women with intact ovaries. Therefore bilateral salpingo-oophorectomy prior to breast cancer is expected to reduce mortality from breast cancer as well as incidence $[2,6]$ in BRCA1 and BRCA2 mutation carriers.

Tumour size has long been recognized as one of the strongest predictors of outcome for patients with invasive breast cancer [7-1 1]. Breast cancer lethality increases with tumour size (although this has not yet been shown in BRCA1 or BRCA2 mutation carriers). We found that prior bilateral oophorectomy was associated with a decreased size of breast tumour in BRCA1 and BRCA2 mutation carriers. Twenty three percent of women with oophorectomy presented with tumours of $1 \mathrm{~cm}$ or less, compared to only four percent of women with intact ovaries. However, we restricted subjects to those with tumours of less than $5 \mathrm{~cm}$, and therefore this study group does not represent all tumours that present in women with BRCA1 or BRCA2 tumours.

BRCA1-associated breast cancers typically present as high-grade tumours [12]. This does not seem to be explained by early age of breast cancer onset in BRCA 1 and BRCA2 mutation carriers [13]. When compared to breast tumours from women of similar ages, BRCA 1 and BRCA2 mutation carriers are shown to be more likely to have higher overall grade tumours [13]. We found that among women with a BRCA2 mutation, those with prior oophorectomy presented with lower grade tumours than those with intact ovaries. We did not observe this effect with BRCAl carriers. High grade is an important index of poor prognosis [14, 15].

The presence of malignant disease in the regional lymph nodes is another independent predictor of breast cancer prognosis $[9,11,16]$. In our study group, women with bilateral oophorectomy were more likely to present with negative lymph nodes. In previous studies, $42 \%$ of BRCA1 mutation carriers presented with positive lymph nodes [17], and $37 \%$ of BRCA2 carriers presented with positive nodes [18]. These frequencies are similar to what we observed in women with intact ovaries in the present study (34\%). However, the proportion of women with positive lymph nodes was lower in women without ovaries (18\%).

It is possible that women who had undergone prophylactic oophorectomy may have been more vigilant in undergoing screening. Breast cancers were diagnosed between 1975 and 2000, therefore we would not expect that many of the subjects would have had access to MRI for breast screening and would have relied on mammography. Our international database of BRCA1 and BRCA2 mutation carriers indicates that only $15 \%$ of all breast cancers were detected by mammography; the great majority (74\%) were detected by breast self-examination (unpublished data). This suggests that vigilant screening would not have affected the results of this study greatly. However, if there was an increase in screening, we may have observed smaller tumours, but screening would not influence the grade of the tumour. Our previous research has shown that nodal status is not positively correlated with tumour size in women with BRCAl mutations [19], and 
therefore we would not expect that women with small tumours would necessarily have negative lymph nodes.

The overall evidence for the importance of preventive oophorectomy for BRCA1 and BRCA2 mutation carriers is increasing. It has previously been established that prophylactic oophorectomy in BRCA1 and BRCA2 mutation carriers prevents the development of breast cancer [2, 6], prevents ovarian cancer [2], and prevents the development of contralateral and ipsilateral breast cancer after initial breast cancer [20]. Bilateral oophorectomy may also offer a beneficial effect on the prognostic factors of breast cancer that develops after oophorectomy and it is expected that survival rates in oophorectomized women will also be higher than those of women who choose to retain their ovaries.

\section{References}

1. Antoniou A, Pharoah PD, Narod S, Risch HA, Eyfjord JE, Hopper $\mathrm{JL}$, Loman N, Olsson H, Johannsson O, Borg A, Pasini B, Radice P, Manoukian S, Eccles DM, Tang N, Olah E, Anton-Culver H, Warner E, Lubinski J, Gronwald J, Gorski B, Tulinius H, Thorlacius S, Eerola H, Nevanlinna H, Syrjakoski K, Kallioniemi OP, Thompson D, Evans C, Peto J, Lalloo F, Evans DG and Easton DF. Average risks of breast and ovarian cancer associated with BRCA1 or BRCA2 mutations detected in case Series unselected for family history: a combined analysis of 22 studies. Am J Hum Genet 2003; 72 (5): $1117-1130$.

2. Rebbeck TR, Lynch HT, Neuhausen SL, Narod SA, Van't Veer L, Garber JE, Evans G, Isaacs C, Daly MB, Matloff E, Olopade OI, Weber BL; Prevention and Observation of Surgical End Points Study Group. Prophylactic oophorectomy in carriers of BRCA1 or BRCA2 mutations. N Engl J Med 2002; 346 (21): 1616-1622.

3. Zavagno G, Meggiolaro F, Pluchinotta A, Bozza F, Favretti F, Marconato R, Geraci G, Nistri R, Fontana P, Sorrentino P, Lumachi F, Toniato A, Rossi CR and Lise M. Influence of age and menopausal status on pathologic and biologic features of breast cancer. Breast 2000; 9 (6): 320-328.

4. Metcalfe KA, Snyder C, Seidel J, Hanna D, Lynch H and Narod $\mathrm{S}$. The use of tamoxifen and other preventive measures among health women who carry a BRCA1 or BRCA2 mutation. Fam Cancer 2004; submitted.

5. Ovarian ablation in early breast cancer: overview of the randomised trials. Early Breast Cancer Trialists' Collaborative Group. Lancet 1996; 348 (9036): 1189-1196.

6. Rebbeck TR, Levin AM, Eisen A, Snyder C, Watson P, CannonAlbright L, Isaacs C, Olopade O, Garber JE, Godwin AK, Daly MB, Narod SA, Neuhausen SL, Lynch HT and Weber BL. Breast cancer risk after bilateral prophylactic oophorectomy in BRCA1 mutation carriers. J Natl Cancer Inst 1999; 91 (17): 1475-1479.

7. Koscielny S, Tubiana M, Le MG, Valleron AJ, Mouriesse H, Contesso $G$ and Sarrazin D. Breast cancer: relationship between the size of the primary tumour and the probability of metastatic dissemination. Br J Cancer 1984; 49 (6): 709-715.

8. Tubiana $M$ and Koscielny $S$. The rationale for early diagnosis of cancer - the example of breast cancer. Acta Oncol 1999; 38 (3): 295-303.

9. Michaelson JS, Silverstein M, Sgroi D, Cheongsiatmoy JA, Taghian A, Powell S, Hughes K, Comegno A, Tanabe KK and Smith B. The effect of tumor size and lymph node status on breast carcinoma lethality. Cancer 2003; 98 (10): 2133-2143.

10. Michaelson JS, Silverstein M, Wyatt J, Weber G, Moore R, Halpern E, Kopans DB and Hughes K. Predicting the survival of patients with breast carcinoma using tumor size. Cancer 2002; 95 (4): $713-723$.

11. Carter CL, Allen C and Henson DE. Relation of tumor size, lymph node status, and survival in 24,740 breast cancer cases. Cancer 1989; 63 (1): 181-7.

12. Foulkes WD, Metcalfe K, Sun P, Hanna WM, Lynch HT, Ghadirian P, Tung N, Olopade OI, Weber BL, McLennan J, Olivotto IA, Begin LR and Narod SA. Estrogen receptor status in BRCA1 and BRCA2related breast cancer: the influence of age, grade, and histological type. Clinical Cancer Research 2004; 10 (6): 2029-2034.

13. Pathology of familial breast cancer: differences between breast cancers in carriers of BRCA1 or BRCA2 mutations and sporadic cases. Breast Cancer Linkage Consortium. Lancet 1997; 349 (9064): 1505-1510.

14. Elston CW and Ellis IO. Pathological prognostic factors in breast cancer. I. The value of histological grade in breast cancer: experience from a large study with long-term follow-up. Histopathology 1991; 19 (5): 403-410.

15. Aaltomaa S, Lipponen P, Eskelinen M, Kosma VM, Mari S, Alhava $\mathrm{E}$ and Syrjanen K. Histological assessment of the prognostic factors in female breast cancer. Oncology 1992; 49 (1): 1-8.

16. Fisher ER, Anderson S, Redmond C and Fisher B. Pathologic findings from the National Surgical Adjuvant Breast Project protocol B-06. 10-year pathologic and clinical prognostic discriminants. Cancer 1993; 71 (8): 2507-2514.

17. Verhoog LC, Brekelmans CT, Seynaeve C, van den Bosch LM, Dahmen G, van Geel AN, Tilanus-Linthorst MM, Bartels CC, Wagner A, van den Ouweland A, Devilee P, Meijers-Heijboer EJ and Klijn JG. Survival and tumour characteristics of breast-cancer patients with germline mutations of BRCA1. Lancet 1998; 351 (9099): 316-321.

18. Verhoog LC, Brekelmans CT, Seynaeve C, Dahmen G, van Geel AN, Bartels CC, Tilanus-Linthorst MM, Wagner A, Devilee P, Halley DJ, van den Ouweland AM, Meijers-Heijboer EJ and Klijn JG. Survival in hereditary breast cancer associated with germline mutations of BRCA2. J Clin Oncol 1999; 17 (1 1): 3396-3402.

19. Foulkes WD, Metcalfe K, Hanna W, Lynch HT, Ghadirian P, Tung N, Olopade O, Weber B, McLennan J, Olivotto IA, Sun P, Chappuis PO, Begin LR, Brunet JS and Narod SA. Disruption of the expected positive correlation between breast tumor size and lymph node status in BRCA 1 -related breast carcinoma. Cancer 2003; 98 (8): 1569-1577.

20. Metcalfe K, Lynch HT, Ghadirian P, Tung N, Olivotto I, Warner E, Olopade OI, Eisen A, Weber B, McLennan J, Sun P, Foulkes WD and Narod SA. Contralateral breast cancer in BRCA1 and BRCA2 mutation carriers. J Clin Oncol 2004; 22 (12): 2328-2335. 